\title{
Primary amelanotic melanoma of the mandibular gingiva
}

\author{
Byeong Jun Kim ${ }^{1}$, \\ Hyeong Seop Kim², \\ Yong Joon Chang', \\ Kee Hwan Kwon', \\ Seong Jin $\mathrm{Cho}^{4}$ \\ ${ }^{1}$ Pop Plastic Surgery Clinic, Seoul; \\ Departments of ${ }^{2}$ Plastic and \\ Reconstructive Surgery, \\ ${ }^{3}$ Otolaryngology-Head and Neck \\ Surgery, and ${ }^{4}$ Pathology, Kangdong \\ Sacred Heart Hospital, Hallym \\ University College of Medicine, Seoul, \\ Korea
}

Oral mucosal melanoma is a very rare type of malignant melanoma, the characteristics of which differ from those of cutaneous melanoma. Primary amelanotic melanoma of the mandibular gingiva, which can invade the mandibular bone, is very rare worldwide. Here, we report a case in which we performed a reconstruction of the mandible and gingiva using the fibula osteocutaneous free flap procedure to treat a patient diagnosed with a primary amelanotic melanoma of the mandibular gingiva. The procedure was successful, and no recurrence was observed 10 months after surgery. Oral mucosal melanoma has a much poorer prognosis and a lower 5-year survival rate than cutaneous melanoma. However, recently, immunomodulatory therapies for mutations in melanocytic lesions have been used effectively to treat the increasing number of patients developing this type of melanoma, thus improving the prognosis of patients with oral mucosal melanoma.

Keywords: Amelanotic / Mandible / Melanoma / Mouth mucosa

\section{INTRODUCTION}

Oral malignant melanoma is a very rare cancer with a poor prognosis. The incidence of melanoma varies by region and race. Oral malignant melanoma accounts for $0.2 \%$ to $8 \%$ of all melanoma cases worldwide [1]. The incidence of melanoma has been increasing in Korea, with 211 patients being treated for melanoma in 2002, 2,567 patients in 2011, and 3,865 patients in 2018 [2]. However, the exact incidence of oral malignant melanoma in Korea is not yet known. The non-pigmented type of oral malignant melanoma is very rare worldwide. Herein, we report the unique case of a primary amelanotic melanoma of the mandibular gingiva.

\footnotetext{
Correspondence: Yong Joon Chang

Department of Plastic and Reconstructive Surgery, Kangdong Sacred Heart

Hospital, Hallym University College of Medicine, 150 Seongan-ro, Gangdong-gu,

Seoul 05355, Korea

E-mail: drjunys@naver.com

Received October 25, 2019 / Revised March 31, 2020 / Accepted April 2, 2020
}

\section{CASE REPORT}

A 63-year-old man presented to the department of dentistry of our hospital with a 1-year history of edema and bleeding of the gingiva around the lesion, along with a 6-month history of unstable teeth and 2-month history of exacerbating pain and bleeding. The patient had no notable medical history, except for a 5 -year history of hypertension. He had quit smoking 5 years ago after having smoked 40 pack years.

At the time of the dental visit, mobility in teeth $31,32,41$, and 42 (International Standards Organization notation) was present, and there was a nodular mass in the gingiva around the teeth. As a result of the excisional biopsy, malignant melanoma, nodular type, was noted. Melanin pigment was not observed upon hematoxylin and eosin (H\&E) staining; therefore, it was confirmed as amelanotic type. Thus, the operation was commissioned to the ear, nose, and throat (ENT).

On physical examination, a $3.0 \times 2.5 \mathrm{~cm}$ sized non-pigmented mass was observed in the mandibular parasymphysis region 
with no pain on palpation (Fig. 1). Preoperative computed tomography (CT), magnetic resonance imaging (MRI), and positron emission tomography-CT did not reveal any lymph node metastasis or distant metastasis. Additionally, no malignant cells were observed in the neck lymph nodes on fine-needle aspiration. However, mandibular bone erosion was observed on CT and MRI and was suspected to be bone invasion due to melanoma (Fig. 2). A preoperative clinical diagnosis of nodular malignant melanoma in the lower gingiva (cT4aN0M0) was made. Physicians in our department and the ENT Department decided on the following treatment plan: (1) wide mass excision with mandibular reconstruction; (2) selective neck dissection at levels I-III; (3) tracheostomy; (4) reconstruction using a

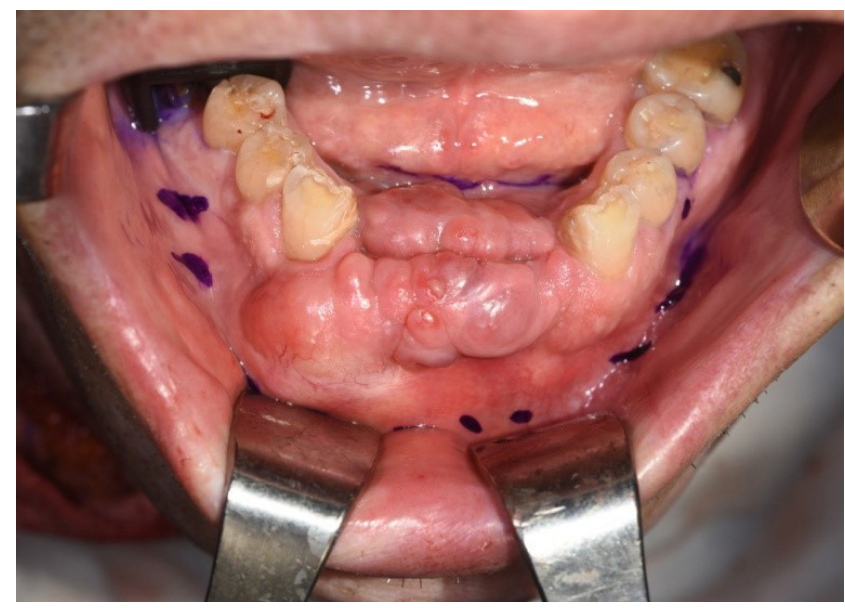

Fig. 1. Preoperative view showing a $3.0 \times 2.5 \mathrm{~cm}$ sized non-pigmented tumor at the mandibular gingiva.

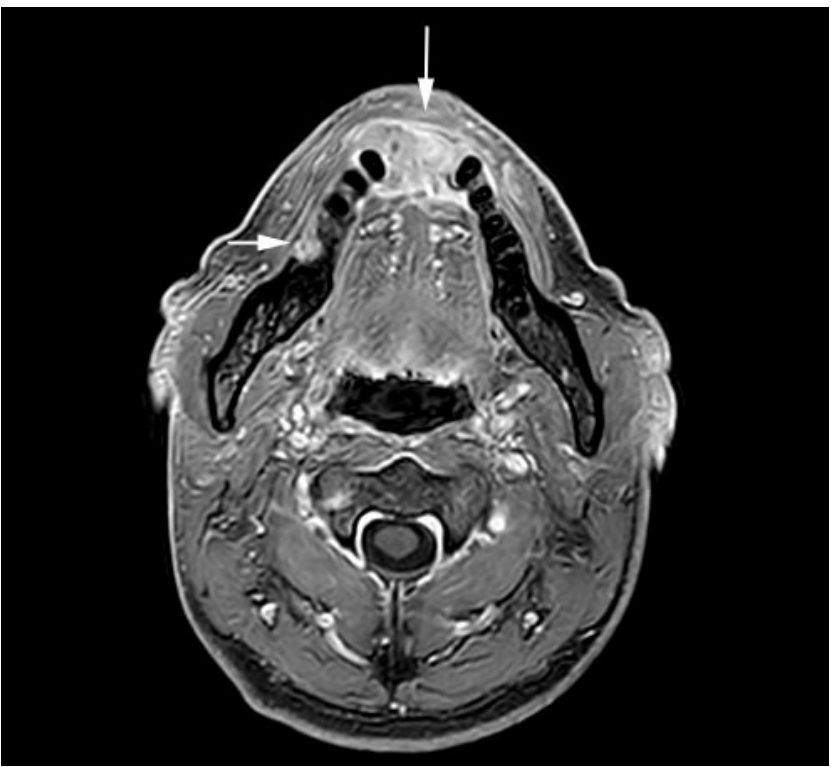

Fig. 2. Magnetic resonance imaging scan showing tumor invasion (arrows) into the mandible. fibula osteocutaneous free flap from the left lower leg and split thickness skin graft; and (5) adjuvant therapy.

The surgical excision margin was $2 \mathrm{~cm}$. A part of the gingiva, vestibule, and floor of the mouth as well as the symphysis and left body of the mandibular bone around the melanoma were removed during surgery by the ENT surgeons (Fig. 3). Simultaneously, we elevated a fibula osteocutaneous free flap by including an 18-cm bone from the left lower leg. The dimensions of the excised mandible were confirmed, the area of the fibula bone placement was designed, and fixation was performed using a reconstruction plate after one-point cutting and angulation (Fig. 4). The skin flap was fixed to the intraoral mucosa by using Vicryl 4-0 sutures. Subsequently, end-to-end microanastomosis

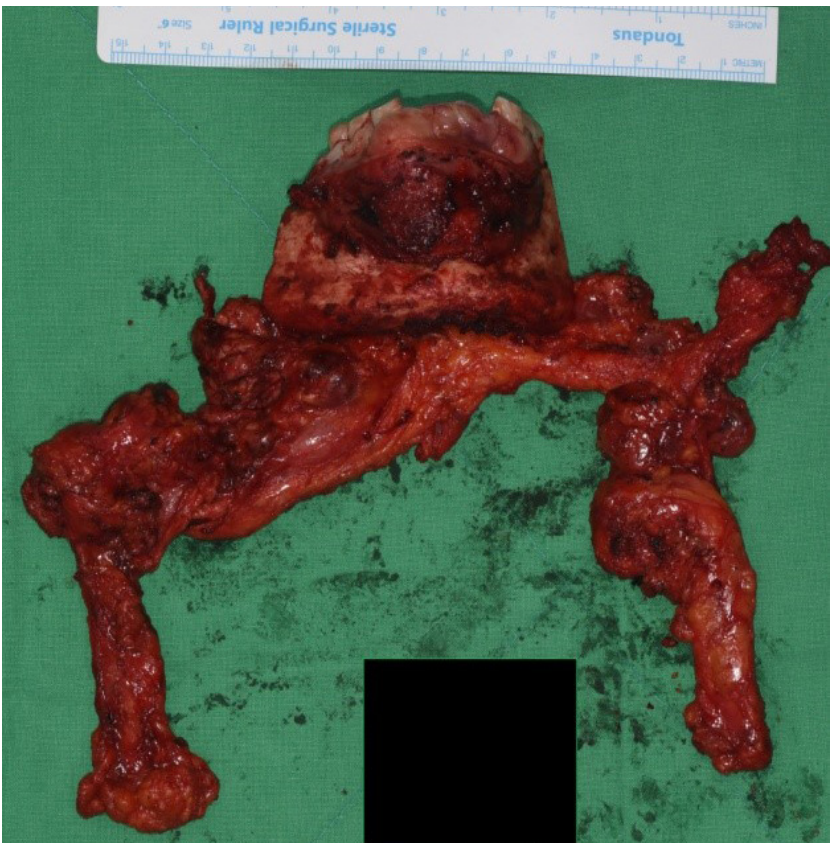

Fig. 3. Excised tumor including the symphysis and left body of the mandible.

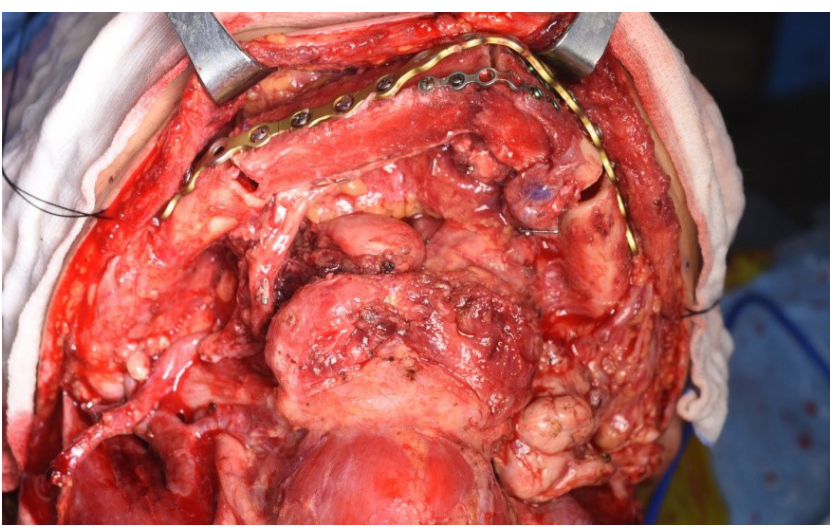

Fig. 4. Intraoperative view showing fixation of the angulated fibula bone to the mandible with plates and screws. 


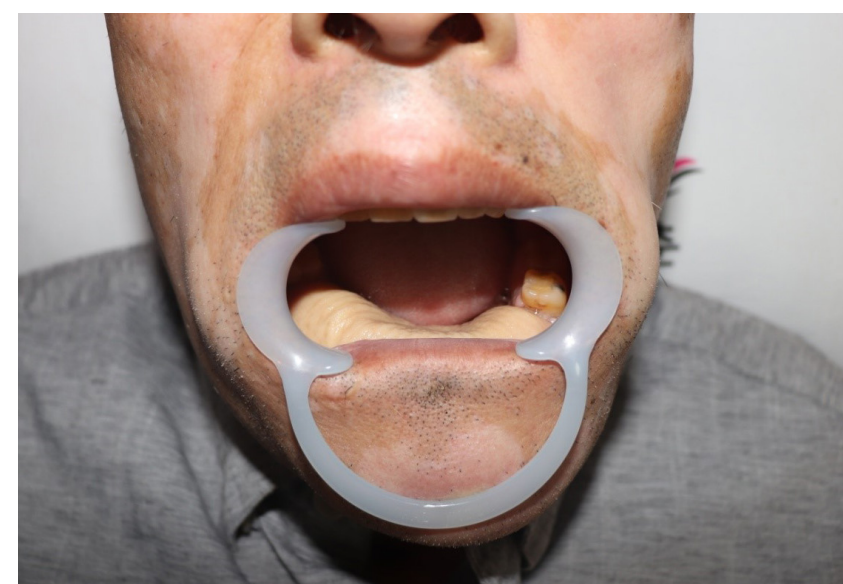

Fig. 5. Follow-up view showing the well maintained intraoral flap 10 months after surgery.

was performed for the peroneal artery and vena comitans of the flap to the facial artery and vein, respectively. The pathological stage of the tumor was confirmed to be pT4aNOM0. One month after surgery, the patient started undergoing radiotherapy $(6,000$ cGy per fraction), with no recurrence observed at 10 months after surgery (Fig. 5).

\section{DISCUSSION}

Melanoma is a malignant tumor that usually occurs on the skin, with varying incidence between races and regions. The incidence of melanoma was 3-5/100,000 people in Mediterranean countries and 12-20/100,000 people in the Nordic countries. However, the incidence of melanoma is increasing gradually [3]. Oral mucosal melanoma is relatively more common among Japanese, African, and American indigenous populations than among Caucasians [4-6]. The incidence of oral mucosal melanoma has not been reported, but based on a report stating that melanoma of the lip comprises $0.8 \%$ of all melanoma cases, the incidence of oral mucosal melanoma in Korea is estimated to be approximately $1 \%$ [7].

Oral mucosal melanoma originates from malignant transformation and clonal expansion of neural crest-derived melanocytes in the basal cell layer of the oral epithelium or the lamina propria of the oral mucosa. The etiology is unknown, and there is no evidence showing that repeated trauma, chronic inflammation, human papilloma virus infection, or tobacco smoking plays an important role in pathogenesis. The only known risk factor is conventional mucosal hyperpigmentation [5].

Oral mucosal melanoma is usually painless and flat, with black, dark brown, or discolored macules or nodules. In rare cases, it may be amelanotic, as in the current case. The difference between pigmented and non-pigmented melanoma is dis-

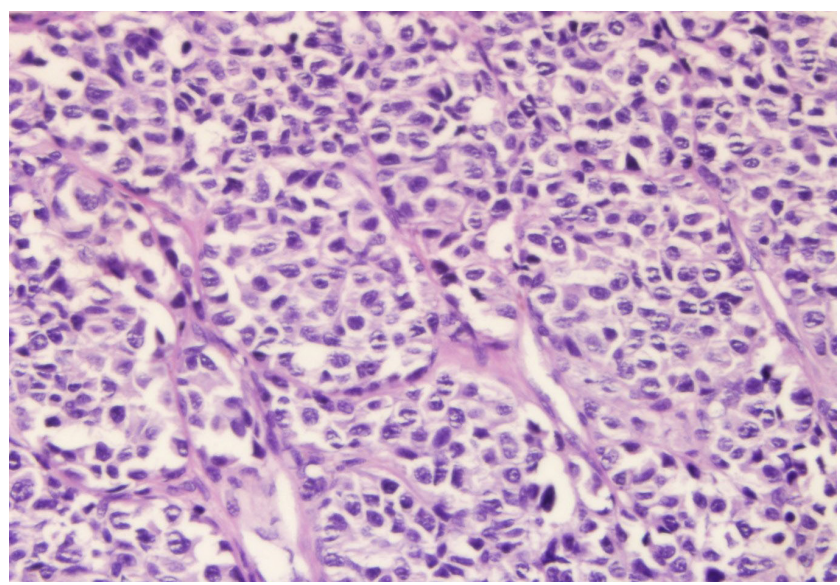

Fig. 6. Histopathologic examination showing pleomorphic cells with granular cytoplasm and hyperchromatic nuclei, but no melanin pigment $(\mathrm{H} \& \mathrm{E}, \times 400)$.

tinguished only by the presence or absence of melanin pigment, and the exact reason for this difference is not yet clear. Oral amelanotic melanoma is sometimes accompanied by erythema or ulceration. Most cases are painless in the early stages with acceptable progress to some extent, although it finally causes pain due to ulceration, growth, or bleeding. Because of these features, amelanotic melanoma has a poorer prognosis owing to the delay in diagnosis and initiation of treatment [8].

A pigmented lesion that cannot be confirmed as benign must be biopsied. If the lesion is large, an incisional biopsy of the most suspicious area is performed. If the lesion is small, an excisional biopsy of the lesion with 1-mm margin is performed. Exfoliative cytology or fine-needle aspiration is contraindicated for primary pigmented lesions. Incisional biopsy must be performed carefully, as it can cause dissemination of malignant cells. In the presence of cervical lymphadenitis, fine-needle aspiration cytology must be performed to determine the presence of malignant cells.

On histological examination, amelanotic melanoma contains spindle-shaped cells with many mitotic figures and, in this case, no cytoplasmic melanin pigmentation (Fig. 6) [9]. These malignant cells have considerable pleomorphism, with large, irregular hyperchromatic nuclei and prominent nucleoli [8]. Oral malignant melanomas can usually be confirmed using H\&Estained sections, but amelanotic melanoma may be mistaken for poorly differentiated adenocarcinoma or poorly differentiated squamous cell carcinoma because it exhibits poorly differentiated pleomorphism and also there is little or no melanin pigment. Therefore, immunohistochemical staining should be added for the diagnosis of melanoma. Useful diagnostic immunohistochemistry markers for amelanotic melanoma include NKI/C-3, human melanoma black-45 (HMB-45), Mart-1 


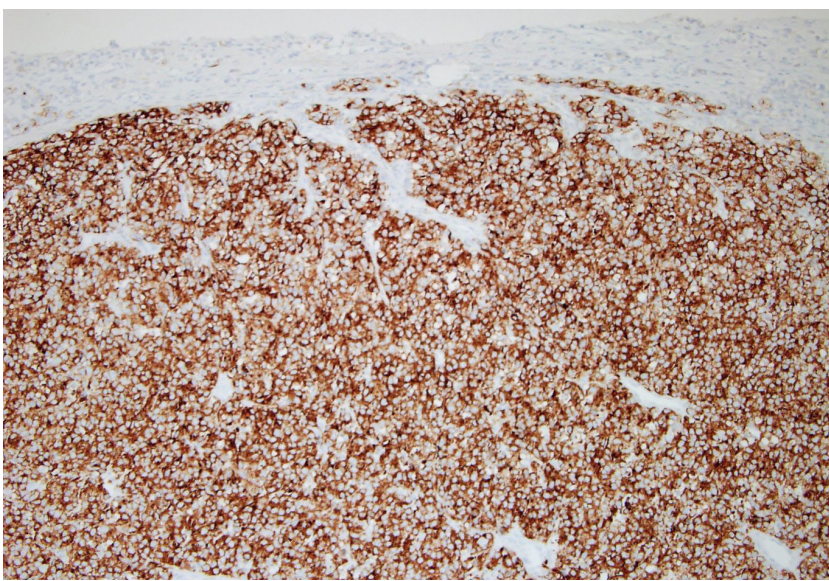

Fig. 7. Immunohistopathologic examination showing tumor invasion into the submucosa (immunostaining for HMB-45, $\times 100$ ).

(Melan-A), S-100 protein, tyrosinase, microphthalmia transcription factor, and vimentin [10]. The current case could not be confirmed to be an amelanotic melanoma despite adding HMB-45 staining, and it was difficult to identify the tumor type on H\&E staining alone (Fig. 7). In oral malignant melanoma cases, the presence of metastasis from the origin is a very important aspect during treatment, as the goal and treatment method are determined considering the presence of metastasis. Primary oral malignant melanoma generally occurs in the hard palate (32\%), maxillary gingiva (16\%), mandibular gingiva (7\%), tongue (7\%), buccal mucosa (7\%), and upper/lower lip (7\%). Secondary or metastatic lesions are more common on the tongue, parotid, and tonsils [11].

Clark classified the stages of cutaneous melanoma into five levels according to the depth of tumor invasion. Breslow then measured the degree of the stratum granulosum invasion (in $\mathrm{mm}$ ) to determine the cutaneous melanoma stage. The Clark criteria for the invasion depth and prognosis are difficult to use for oral malignant melanoma owing to the lack of histological structures similar to the papillary and reticular dermis [12].

Ballantyne classified mucosal melanoma into three disease types in 1970: stage I, clinically localized; stage II, regional lymph node; and stage III, distant [12]. This classification is simple and applicable for all primary mucosal melanomas, but it is limited by the fact that the invasion depth or local tumor extension is not considered. Furthermore, this classification over-emphasizes regional spread, which is relatively uncommon in primary mucosal melanomas, thereby offering limited prognostic information regarding the majority of patients with stage I disease (75\%-95\%). To overcome these limitations, Prasad et al. [13] devised a microstaging system based on the invasion of mucosal tissue compartments: level 1, in situ disease; level 2, superficially invasive melanoma invading up to the lamina pro- pria; and level 3, deeply invasive melanoma invading the muscle, bone, or cartilage. Prasad et al. reported that the disease-specific survival (DSS) rates were significantly different considering the level, with DSS rates of 75\%, 52\%, and 23\% for levels I, II, and III, respectively. However, the limitation of this microstaging technique is that it requires histological examination and can only be determined after surgical tumor excision, except in cases with radiologically confirmed deep tumor invasion.

The American Joint Committee on Cancer did not have staging guidelines for mucosal melanoma until the 7th edition, wherein T3 and T4 designations that emphasize the extent and size of the primary tumor were added as prognostic factors for the area of origin.

Surgical excision is the treatment of choice for oral mucosal melanoma, similar to the method used for treating other cancers. Oral mucosal melanoma is treated with wide excision based on the same criteria used for cutaneous melanoma. However, if bone invasion is observed, as noted in the current case, additional ostectomy is needed. In case of mandibular involvement, a chimeric flap including the bone is necessary for reconstruction after ostectomy.

Over the past few decades, neither cytotoxic chemotherapy nor combination regimens have been able to improve the overall survival of patients with advanced and metastatic melanoma. However, medical treatment has vastly improved, and researchers now have a much better understanding of the molecular pathophysiology of melanoma. In particular, immunomodulatory therapies targeting mutations in melanocytic lesions have been significant recent breakthroughs. The U.S. Food and Drug Administration has recently approved the use of ipilimumab (2011), peginterferon alfa-2b (2011), vemurafenib (2011), dabrafenib (2013), trametinib (2013), pembrolizumab (2014), and nivolumab (2014). Among cancer immunotherapy agents, immune checkpoint inhibitors that modulate programmed cell death- 1 and its ligand have been demonstrated to be effective for treating progressive cutaneous melanoma [14], although further study is needed to determine their efficacy for oral mucosal melanoma. In the current case, pathologically confirmed pT4aN0M0, only postoperative radiotherapy was performed according to the National Comprehensive Cancer Network guidelines. In consultation with the department of hematology and oncology, cancer immunotherapy should be performed in the future if needed.

Oral mucosal melanoma is more aggressive than cutaneous melanoma. The 5-year survival rate of cutaneous melanoma patients is $43 \%-44 \%$, whereas the survival of oral mucosal melanoma patients is only $9.4 \%-15.6 \%$ even after radical treatment. Survival decreased with the increasing duration from di- 
agnosis to treatment [9]. The reasons for poorer prognosis include late diagnosis, difficulty in performing curative surgery because of anatomical limitations, faster invasion in mucosal tumors, vascularity of the oral mucous membrane, and the presence of a thinner mucous membrane than the skin owing to the histological absence of a reticular dermis and thinner lamina propria because of a thin papillary dermis [11].

Recently, Wermker et al. [15] analyzed the data of 42 patients with primary mucosal melanoma of the head and neck and determined a few prognostic factors as follows: age $>70$ years, the presence of distant metastasis, and lymphovascular invasion, all of which were important factors associated with unfavorable outcomes and decreased DSS. Oral mucosal melanoma is rarer but much more aggressive than cutaneous melanoma, with a poorer prognosis. In this case report, we highlight the potential for local spread of amelanotic mucosal melanoma and describe the characteristics of mucosal melanoma. We conclude that the treatment used in the current case, along with recent breakthroughs in medical treatment, might improve the survival rate of mucosal melanoma patients.

\section{NOTES}

\section{Conflict of interest}

No potential conflict of interest relevant to this article was reported.

\section{Ethical approval}

The study was approved by the Institutional Review Board of Kangdong Sacred Heart Hospital (IRB No. 2019-10-010) and performed in accordance with the principles of the Declaration of Helsinki. Written informed consent was obtained.

\section{Patient consent}

The patient provided written informed consent for the publication and the use of his images.

\section{ORCID}

Byeong Jun Kim https://orcid.org/0000-0002-8539-842X

Hyeong Seop Kim https://orcid.org/0000-0003-4037-2805

Yong Joon Chang https://orcid.org/0000-0003-4966-6543

Kee Hwan Kwon https://orcid.org/0000-0002-5458-6903

Seong Jin Cho https://orcid.org/0000-0002-3491-6848

\section{REFERENCES}

1. Chiu NT, Weinstock MA. Melanoma of oronasal mucosa: population-based analysis of occurrence and mortality. Arch
Otolaryngol Head Neck Surg 1996;122:985-8.

2. National Health Insurance Service. National Health Insurance Statistical Yearbook 2002, 2011, and 2018 [Internet]. Wonju: National Health Insurance Service [cited 2020 Apr 28]. Available from: http://lib.nhis.or.kr/.

3. MacKie RM, Bray C, Vestey J, Doherty V, Evans A, Thomson D, et al. Melanoma incidence and mortality in Scotland 19792003. Br J Cancer 2007;96:1772-7.

4. Shiga K, Ogawa T, Kobayashi T, Ueda S, Kondo A, Nanba A, et al. Malignant melanoma of the head and neck: a multi-institutional retrospective analysis of cases in northern Japan. Head Neck 2012;34:1537-41.

5. Patrick RJ, Fenske NA, Messina JL. Primary mucosal melanoma. J Am Acad Dermatol 2007;56:828-34.

6. Smith MH, Bhattacharyya I, Cohen DM, Islam NM, Fitzpatrick SG, Montague LJ, et al. Melanoma of the oral cavity: an analysis of 46 new cases with emphasis on clinical and histopathologic characteristics. Head Neck Pathol 2016;10:298-305.

7. Oh CM, Cho H, Won YJ, Kong HJ, Roh YH, Jeong KH, et al. Nationwide trends in the incidence of melanoma and nonmelanoma skin cancers from 1999 to 2014 in South Korea. Cancer Res Treat 2018;50:729-37.

8. Lopez F, Rodrigo JP, Cardesa A, Triantafyllou A, Devaney KO, Mendenhall WM, et al. Update on primary head and neck mucosal melanoma. Head Neck 2016;38:147-55.

9. Ascierto PA, Accorona R, Botti G, Farina D, Fossati P, Gatta G, et al. Mucosal melanoma of the head and neck. Crit Rev Oncol Hematol 2017;112:136-52.

10. Ohsie SJ, Sarantopoulos GP, Cochran AJ, Binder SW. Immunohistochemical characteristics of melanoma. J Cutan Pathol 2008;35:433-44.

11. Lamichhane NS, An J, Liu Q, Zhang W. Primary malignant mucosal melanoma of the upper lip: a case report and review of the literature. BMC Res Notes 2015;8:499.

12. Ballantyne AJ. Malignant melanoma of the skin of the head and neck: an analysis of 405 cases. Am J Surg 1970;120:425-31.

13. Prasad ML, Patel SG, Huvos AG, Shah JP, Busam KJ. Primary mucosal melanoma of the head and neck: a proposal for microstaging localized, stage I (lymph node-negative) tumors. Cancer 2004;100:1657-64.

14. Knackstedt T, Knackstedt RW, Couto R, Gastman B. Malignant melanoma: diagnostic and management update. Plast Reconstr Surg 2018;142:202e-216e.

15. Wermker K, Brauckmann T, Klein M, Habfeld S, Schulze HJ, Hallermann C. Prognostic value of S100/CD31 and S100/ podoplanin double immunostaining in mucosal malignant melanoma of the head and neck. Head Neck 2015;37:1368-74. 